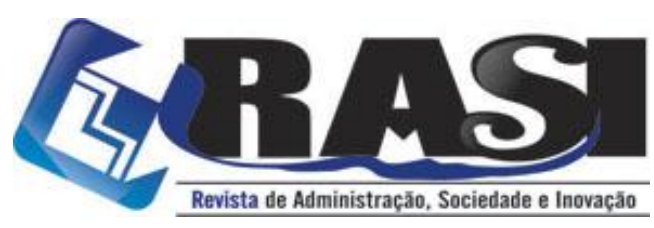

http://www.rasi.uff.br

RASI, Volta Redonda/RJ, v. 4, n. 2, pp. 204-224, jul./dez. 2018

\title{
O Uso da Accountability Theory em Pesquisas sobre Transparência Governamental: Abordagens e Perspectivas
}

\author{
Rodrigo Hickmann Klein (PUC/RS) - rodrigo.hickmann@acad.pucrs.br \\ Deisy Cristina Barbiero Klein - deisy.klein@gmail.com \\ Edimara Mezzomo Luciano (PUC/RS) - eluciano@pucrs.br
}

RESUMO: No desenvolvimento de uma cultura de transparência, responsabilização e qualidade do gasto público, a responsabilização almejada pode ser alcançada pelos componentes e mecanismos da Accountability Theory (AT), que abrange processos no qual os indivíduos têm a obrigação potencial de explicar suas ações a outros indivíduos, os quais têm o direito de julgar as ações e administrar consequências positivas ou negativas em resposta a essas ações. Por intermédio de uma revisão sistemática da literatura, a presente pesquisa identificou abordagens e estratégias de pesquisa sobre AT. Dentre os usos da AT, destacou-se o reiterado uso de mecanismos de prestação de contas e responsabilização na avaliação de desempenho de funcionários, públicos ou privados, e a relação do uso eficaz desses mecanismos à melhoria do desempenho. As expectativas de responsabilização tornam os funcionários mais dispostos a assumir e apoiar ações orientadas para a conformidade, quando visa à prevenção, detecção e punição de violações de leis e regras. Os resultados demonstraram uma potencial área de pesquisa empírica sobre o uso da AT, abrangendo a transparência e o combate à corrupção, pois a prestação de contas, e a posterior responsabilização dos agentes públicos envolvidos, são inerentes à legislação sobre controle dos gastos e ações governamentais.

PALAVRAS-CHAVE: Accountability Theory; transparência; responsabilização; prestação de contas; governo.

\section{The Use of Responsibility Research Theory on Government Transparency: Approaches and Perspectives}

ABSTRACT: In developing a culture of transparency, accountability, and quality of public spending, accountability might be achieved by the Accountability Theory (AT) components and mechanisms, which encompass processes in which individuals have the potential obligation to explain their actions to other individuals who have the right to judge actions and administer positive or negative consequences in response to such actions. Through a systematic review of literature, the present research identified approaches and strategies of researches on AT and how this theory is used in researches on the transparency of government actions and expenditures. Among the uses of AT there is repeated use of mechanisms of accountability in the evaluation of the performance of public or private employees, and the relation of the effective use of these mechanisms to improve performance. Expectations of accountability make employees more willing to assume and support compliance-oriented actions when they aim to prevent, detect and punish violations of laws and rules. The results demonstrated a potential area of empirical research on the use of AT, encompassing transparency and the fight against corruption, since subsequent accountability of the public agents involved are inherent to the legislation on control of government spending and actions.

KEYWORDS: Accountability Theory; transparency; government.

\section{Universidade \\ Vederal Fluminense}

R. Desembargador Ellis Hermydio Figueira, 783, Bloco A, sl. 218, Aterrado.

27213-415 - Volta Redonda, RJ - Brasil www.uff.br

Copyright (C) 2018 RASI. Todos os direitos, até mesmo de tradução, são reservados. É permitido citar parte de artigos sem autorização prévia, desde que seja identificada a fonte 


\section{O Uso da Accountability Theory em Pesquisas sobre Transparência Governamental: Abordagens e Perspectivas}

\section{Introdução}

As relações entre a informação, a transparência e a democracia são fundamentais e básicas (Harrison et al., 2012). A informação é essencial para o desenvolvimento de competências democráticas fundamentais, como por exemplo, a formulação de preferências e opiniões, a conjuntura de hipóteses e a participação na tomada de decisões. Sem essas competências, é negada a voz ao cidadão e o exercício de seus direitos. Conforme De Ferranti et al. (2009), a transparência, quando aplicada à área governamental, diz respeito à disponibilidade pública e oportuna, com qualidade, abrangente e relevante, de informações confiáveis sobre as atividades do governo, considerado essencial no fornecimento de uma fonte contínua de informações para a aprovação do governante pelo cidadão. Para Harrison et al. (2012), abrange a divulgação voluntária e rotineira dos orçamentos, auditorias, políticas e ações executivas, ampliando as exigências sobre os serviços públicos que são prestados pelo governo ao cidadão, gerando pressão para melhorar o desempenho dos entes públicos, fornecendo ao cidadão um feedback contínuo e permitindo avaliações mais abrangentes dos serviços governamentais. Sol (2013) acrescenta que a transparência ajuda a responsabilizar os funcionários do governo por suas ações e omissões.

Nesse sentido, a transparência aumenta a exposição das operações do governo ao exame minucioso dos vários componentes do sistema político (Stamati et al., 2015) e a probabilidade da corrupção ser detectada (Andersen, 2009). Para Murillo (2015) a accountability está intimamente ligada à transparência, como um importante redutor dos níveis de corrupção, através da responsabilização dos agentes públicos. Já para Al-Jamal e Abu-Shanab, (2016) a transparência é considerada o precursor da accountability.

Para Pinho e Sacramento (2009) a accountability envolve responsabilidade objetiva e subjetiva, controle, transparência, obrigação de prestação de contas e justificativas para as ações que foram ou deixaram de ser empreendidas, bem como, recompensas e sanções. Os autores destacam que a transparência das ações governamentais, como uma dimensão da accountability, é capaz de despertar mais condições de confiança nos governados perante os governantes, pois a transparência é vista como capaz de contribuir para reduzir à corrupção no espaço público e concomitantemente estabelece relações entre o Estado e a sociedade civil mais democrática (Pinho \& Sacramento, 2009).

No entanto, segundo Raupp e Pinho (2013), a accountability pode possuir uma abordagem ainda mais ampla, que considera os mecanismos de controle formalizados e institucionalizados, capazes de exigir a responsabilização dos agentes públicos pelos atos praticados; e mecanismos informais, como o controle exercido pela imprensa e pela sociedade civil. Dessa forma, a execução da accountability requer capacidade de resposta dos agentes públicos, por intermédio da capacidade de informar sobre os atos, depende também da capacidade de sofrer sanções e perda de poder para aqueles que violaram os deveres, que depende da capacidade de punição (Raup \& Pinho, 2013).

Entretanto, para Akutsu e Pinho (2002) o conceito de accountability abrange duas partes, a primeira parte delega responsabilidade para que a segunda parte proceda à gestão dos recursos. Concomitantemente, gera a obrigação dos administrares dos recursos de prestar contas de sua gestão, demonstrando o bom uso desses recursos.

No entanto, Campos (1990) menciona que somente a partir da organização de cidadãos vigilantes e conscientes de seus direitos haverá condição para a accountability, pois 
não haverá tal condição enquanto o povo se definir como tutelado e o Estado como tutor. Portanto, a accountability somente pode ser garantida pelo exercício da cidadania ativa, não por cidadãos individualmente, mas pela cidadania organizada (Campos, 1990).

Schillemans et al. (2013) argumentam que a accountability não é apenas benéfica aos cidadãos, mas também é potencialmente vantajosa para as próprias organizações públicas, pois ao fornecer a prestação de contas de suas ações, as instituições governamentais podem demonstrar sua legitimidade. Segundo os autores, a accountability é um espelho para a organização pública, ao refletir sobre suas ações passadas, as organizações públicas podem aprender com seus erros e sucessos, e assim melhorar seu desempenho no futuro, o que novamente pode aumentar seu apoio pelos cidadãos.

Segundo Janssen (2011), as iniciativas de Dados Abertos Governamentais (DAG) podem ajudar os cidadãos a aprender sobre as atividades governamentais, melhorar a accountability governamental e permitir que os cidadãos participem do processo político. Para Lourenço (2015), o DAG promove a transparência através da publicação de dados do governo, possibilitando ao cidadão ver o que o governo faz, permitindo a responsabilização dos agentes públicos e dos representantes eleitos por suas ações, omissões e decisões tomadas, além de divulgar informações governamentais que podem ser reutilizadas e proporcionem valor social e econômico. Höffner et al.(2016) acrescenta que os dados abertos de gastos do governo têm o poder de reduzir a corrupção, aumentando a accountability e fortalecendo a democracia, porque os eleitores podem tomar decisões estando melhor informados, e um público informado e confiante também fortalece o próprio governo, pois é mais provável que se comprometa com iniciativas governamentais.

Accountability é um termo comumente utilizado na literatura científica, porém seu uso na teoria adotada na presente pesquisa surge por intermédio dos estudos de P. E. Tetlock, que desenvolveu os conceitos e mecanismos iniciais da denominada Accountability Theory (Lerner \& Tetlock, 1999; Vance et al., 2013) em dois artigos seminais: Tetlock (1983a) e Tetlock (1983b). Os conceitos apresentados nesses artigos foram evoluindo por meio de artigos posteriores, como Tetlock (1985), Tetlock (1987), Tetlock et. al. (1989), Tetlock e Boettger (1989) e Tetlock e Boettger (1994). Posteriormente, J. S. Lerner trabalhou junto com P.E. Tetlock em um artigo (Lerner \& Tetlock, 1999) para desenvolver o que é referido atualmente como Accountability Theory (Vance et al. 2015). Nessa teoria, a accountability tem dois enfoques mais comuns (Vance et al., 2013):

a) Como uma virtude: a accountability é vista como uma qualidade em que uma pessoa demonstra a vontade de aceitar a responsabilidade, tornando-se uma característica desejável em funcionários públicos, agências governamentais ou empresas. Assim, neste uso, a accountability é uma característica positiva;

b) Como um mecanismo: a accountability é vista como um processo em que um indivíduo tem uma obrigação de explicar suas ações e decisões a outros indivíduos que tem o direito de julgá-las, bem como submeter esse indivíduo a potenciais consequências por suas ações e decisões. Neste enfoque a accountability incide sobre o processo de prestação de contas e responsabilização.

Segundo Vance et al. (2015), a responsabilização pode ser obtida pelos componentes e mecanismos de responsabilização da Accountability Theory (AT), que abrange o processo no qual os indivíduos têm a obrigação potencial de explicar suas ações a outros indivíduos, os quais têm o direito de julgar as ações e administrar consequências positivas ou negativas em resposta a essas ações. Para Vance et al. (2013), a AT tem quatro componentes fundamentais. $\mathrm{O}$ primeiro deles é a identificabilidade, que é a crença de um indivíduo em ser identificado. O 
segundo é a expectativa de avaliação, que sucede da crença de um indivíduo de que seu desempenho será avaliado por outros, de acordo com algumas regras básicas normativas e com consequências implícitas. O terceiro componente é a consciência de monitoramento, um estado da cognição ativo, no qual um indivíduo considera que o trabalho, relacionado a um sistema, é monitorado. Por fim, o quarto componente é a presença social, que é a consciência da existência de outros usuários de um determinado sistema.

Entretanto, para que o uso desses componentes e mecanismos de responsabilização possa evoluir em pesquisas futuras sobre a AT na transparência de ações e dados governamentais, é necessário conhecer quais são as abordagens e estratégias de pesquisa dessa teoria. Deste modo, a presente pesquisa visa responder a seguinte questão de pesquisa: quais são as abordagens e estratégias de pesquisa sobre AT e como essa teoria é utilizada em pesquisas sobre transparência e dados abertos governamentais?

Portanto, o presente estudo tem como objetivo revisar e categorizar as pesquisas existentes sobre AT e sua relação com códigos axiais definidos a priori, relacionados à transparência e dados abertos governamentais, identificando quais abordagens estão sendo adotadas, quais são as estratégias de pesquisa utilizadas, como evoluiu o conceito, quais os artigos mais referenciados e como essa teoria se relaciona com a transparência e com os dados abertos governamentais. Para atender a esse objetivo, a presente pesquisa realizou uma Revisão Sistemática da Literatura (RSL) sobre essa teoria, com uma posterior categorização das pesquisas analisadas.

O restante do presente estudo está organizado de acordo com o recomendado por Cooper et al. (2009) para uma Revisão Sistemática da Literatura, conforme segue: na segunda seção é apresentado o método de pesquisa, descrevendo a sistemática da revisão da literatura, incluindo: a) como as bases de dados foram pesquisadas; b) anos pesquisados; c) termos de busca; e) critérios de inclusão e exclusão, com fundamentação teórica e empírica; d) sistemáticas tomadas para minimizar viés e erros no processo de seleção do estudo; e) descrição das fases de identificação, rastreio, elegibilidade e inclusão, com o número de estudos incluídos e excluídos em cada fase e as razões. Na terceira seção é apresentada a análise dos resultados, no qual são descritas as características dos estudos abrangidos, englobando a avaliação crítica e sistemática. Por fim, na quarta seção são descritos os resultados e as conclusões da revisão no contexto da teoria, vinculando conclusões de forma explícita e intuitiva às evidências revisadas.

\section{Procedimento Metodológico}

Para atingir os objetivos estabelecidos, foi realizada uma Revisão Sistemática da Literatura. As revisões sistemáticas visam abordar problemas de identificação, avaliação crítica e integração das conclusões de todos os estudos individuais relevantes e de elevada qualidade que abordam uma ou mais questões de investigação. Uma revisão sistemática pode atingir a maioria ou todos os seguintes objetivos (Baumeister \& Leary, 1997; BEM, 1995): a) estabelecer até que ponto a pesquisa existente progrediu para esclarecer um problema particular; b) identificar relações, contradições, lacunas e inconsistências na literatura, explorando razões, propondo uma nova conceitualização ou teoria que explique a inconsistência; c) formular declarações gerais ou uma conceituação abrangente; d) comentar, avaliar, ampliar ou desenvolver a teoria; e) fornecer implicações para a prática e políticas e f) descrever orientações para pesquisas futuras.

Conforme as orientações de Cooper et al. (2009), a análise dos artigos foi composta por seis fases, realizadas entre janeiro e março de 2017. Na primeira delas, foi realizada a 
consulta nos Bibliographic DataBases (BD - base de dados bibliográficas) Scopus, Web of Science e ScienceDirect. As bases de dados bibliográficas Scopus, Web of Science foram escolhidas por serem consideradas as bases mais importantes conforme Wang e Waltman (2016) e a ScienceDirect com maior resultado conforme Tober (2011).

Na Tabela 1, constam o termo pesquisado ("ACCOUNTABILITY THEORY"), as bases de dados bibliográficas, os critérios e a quantidade de artigos encontrados (124 artigos), indexados até 02/03/2017.

Tabela 1 - Termo pesquisado e bases de dados bibliográficas utilizadas.

Termo pesquisado: "ACCOUNTABILITY THEORY"

\begin{tabular}{c|c|c}
\hline Base de Dados & Critério de pesquisa & $\begin{array}{c}\mathbf{N}^{\circ} \text { Artigos } \\
\text { Encontrados }\end{array}$ \\
\hline SCOPUS (2017) & $\begin{array}{c}\text { In: Article Title, Abstract, Keyword; } \\
\text { Document type: Article; } \text { Subject Area: all } \\
\text { science; } \text { All years. }\end{array}$ & 35 \\
\hline $\begin{array}{c}\text { SCIENCEDIRECT } \\
\text { (2017) }\end{array}$ & $\begin{array}{c}\text { In: Abstract, Title, } \text { Keyword; } \text { Refine: } \text { Journal } \\
\text { All Sciences; } \text { All years; } \text { Except: Article status } \\
\text { "Corrected Proof". }\end{array}$ & 63 \\
\hline $\begin{array}{c}\text { WEB OF SCIENCE } \\
\text { (2017) }\end{array}$ & $\begin{array}{c}\text { Topic: "ACCOUNTABILITY THEORY" } \\
\text { OR Title: "ACCOUNTABILITY THEORY" } \\
\text { Document type: ARTICLE; } \text { All years. }\end{array}$ & 26 \\
\hline
\end{tabular}

Fonte: Dados da Pesquisa (2017).

$\mathrm{Na}$ segunda fase, foi realizada a remoção dos artigos repetidos. Nesta fase, também foram removidos os artigos oriundos de eventos e de conferências, remanescendo 101 artigos. $\mathrm{Na}$ terceira fase foi feita a verificação dos artigos, com base no conteúdo do artigo, para confirmar se os artigos utilizam conceitos da Accountability Theory (AT). Os artigos que não atendiam a esse critério foram retirados da seleção. Em relação à linguagem, foram mantidos somente artigos no idioma inglês. Como resultado desta fase, permaneceram 36 artigos que foram analisados por esta pesquisa. A quantidade de artigos desconsiderados foi acentuada, pois o mecanismo de pesquisa do ScienceDirect não considerou o uso de aspas no termo composto de pesquisa "accountability theory".

A quarta e quinta fases visaram sintetizar enfoques de utilização da AT. Desta forma, na quarta fase foram analisados os termos comuns dentro de cada definição sobre AT, através da análise de conteúdo, obedecendo às etapas de redução, de exibição, de conclusão e verificação dupla, isoladamente (double bind), conforme a recomendação de Flick (2009). Na quinta fase foram identificados os termos mais abordados nos artigos, para isso foi utilizada a funcionalidade Word Cloud do NVIVO 11.

A sexta fase visou à categorização dos artigos. Para tanto, foram realizadas buscas nos artigos, por intermédio da ferramenta de search do software MAXQDA 12, utilizando os códigos de cada categoria axial de enfoque, produzindo uma classificação dos artigos em seis categorias. Os códigos axiais definidos a priori (Bardin, 2011) foram: transparency, government, corruption, participation, open data, open government, também foram pesquisados termos correlatos como corrupt, transparenc e particip. Os códigos axiais utilizados foram obtidos das pesquisas de Hossain et al. (2016) e Attard et al. (2015), que citaram em conjunto esses códigos como categorias analisadas nas respectivas pesquisas, relacionados direta e indiretamente com a prestação de contas e responsabilização. Por intermédio dos excertos obtidos pelo MAXQDA, os artigos foram analisados novamente e 
designados à respectiva categoria axial, através da análise de conteúdo, obedecendo às etapas de redução, de exibição, de conclusão e verificação dupla, isoladamente (double bind) (Flick, 2009).

\section{Análise dos Resultados}

A análise dos resultados está subdivida em três partes. Na seção 3.1, é realizada a análise descritiva dos artigos abordando as publicações por ano, principais autores e periódicos que abordam a AT, abrangendo os artigos que estão sendo referenciados dentre os coletados e fora desde escopo, além dos principais artigos sobre AT. Na seção 3.2, são analisadas as definições sobre AT e os principais termos utilizados, principais usos da AT, e o uso com relação às ações, decisões e dados governamentais, bem como uma síntese dos principais conceitos apresentados pelos artigos. Ao final, na seção 3.3, é explorada a proposição de um possível enfoque para a ampliação de pesquisas sobre o uso AT na área governamental.

\subsection{Análise descritiva dos resultados}

Conforme ilustrado no Gráfico 1, não foram encontradas pesquisas anteriores a 1979, apesar de não ter sido estabelecido um limite de anos dentre os critérios de pesquisa descritos na Tabela 1. O termo accountability, embora utilizado em pesquisas científicas anteriores a 1979, não foi referenciado como um conceito da Accountability Theory, que é o foco da presente pesquisa.

Os resultados demonstram um aumento significativo de artigos nos últimos anos, demonstrando o incremento do interesse científico pelo tema. O ano de 2017 apresenta uma queda, porém a coleta considerou apenas os artigos indexados até 02/03/2017.

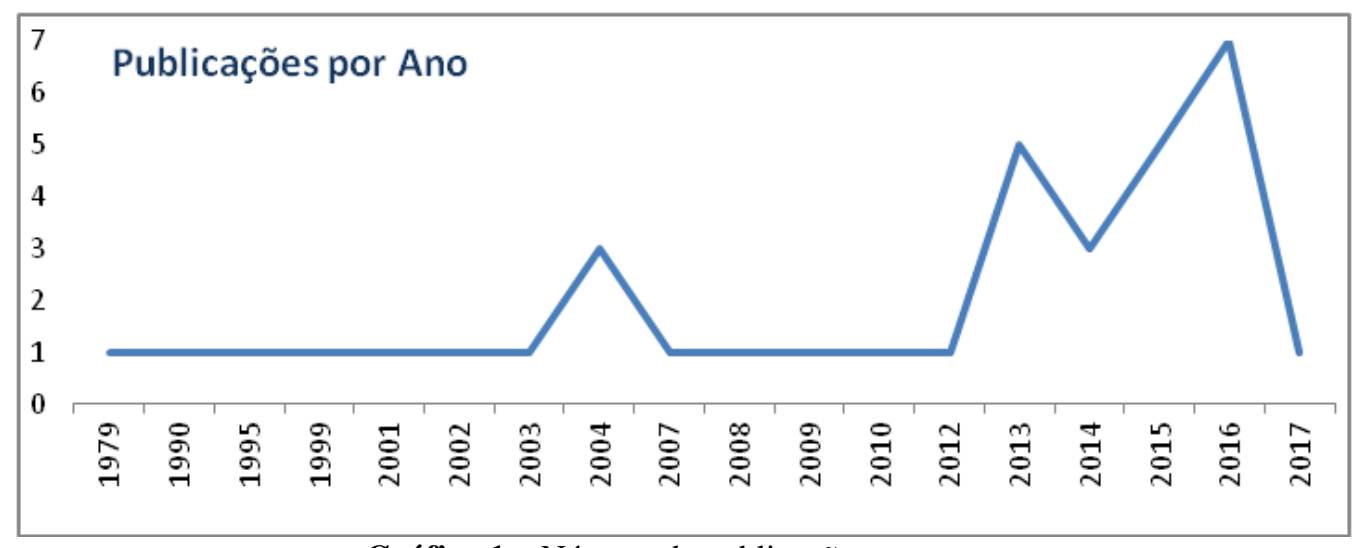

Gráfico 1 - Número de publicações por ano. Fonte: Dados da Pesquisa (2017).

No Gráfico 2, são identificados os periódicos com número de publicações sobre AT superiores a um artigo. O periódico Human Resource Management Review se destaca dos demais, somando seis publicações, destacando a maior utilização da Accountability Theory em pesquisas da área de gestão de pessoas, abrangendo principalmente como a prestação de contas influência no desempenho organizacional dos indivíduos sujeitos à responsabilização. 


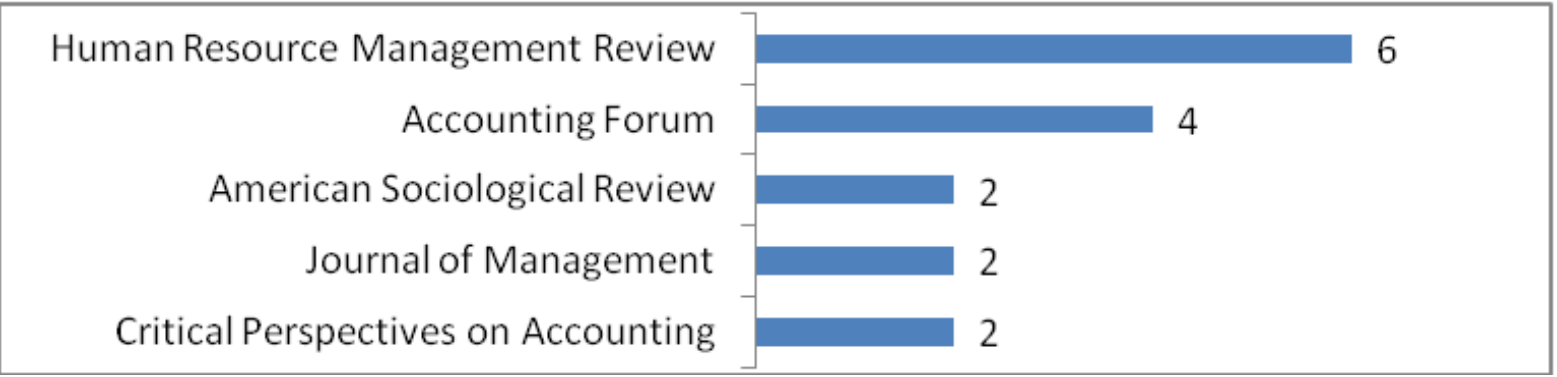

Gráfico 2 - Quantidade de artigos por periódico científico.

Fonte: Dados da Pesquisa (2017).

Na Figura 1, são exibidas as citações dentre os 36 artigos selecionados para análise nesta pesquisa. O artigo mais citado dentre os selecionados é o artigo Klimoski e Inks (1990), que aborda a AT aplicada ao contexto da avaliação de desempenho de funcionários em organizações. O segundo mais citado é Vance et al. (2015) que utilizaram a AT para desenvolver quatro artefatos de interface para um sistema, com o objetivo de aumentar as percepções de responsabilidade dos usuários dentro desse sistema e, por sua vez, diminuir as violações da política de acesso.

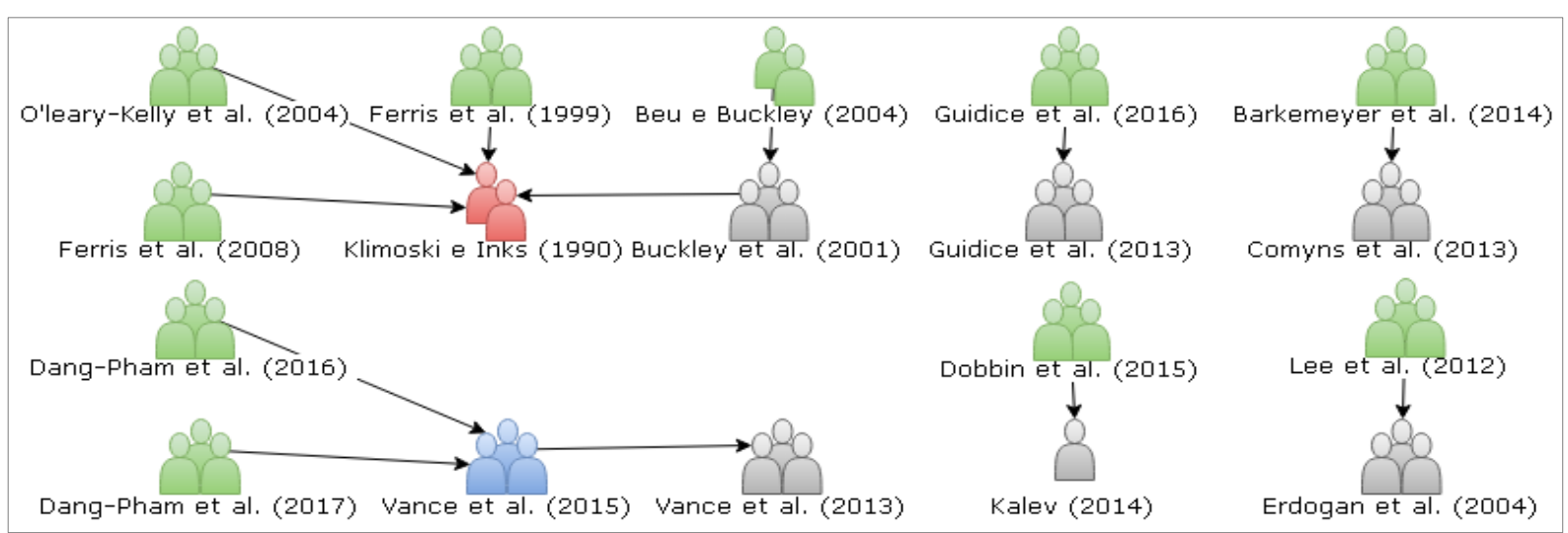

Figura 1 - Citações dentre os 36 artigos selecionados.

Fonte: Dados da Pesquisa (2017).

Na Tabela 2, são exibidos os 10 artigos com maior número de citações em geral de acordo com a respectiva referência à base de dados (BD), demonstrando que as pesquisas que utilizam AT, são bem referenciadas por demais artigos que não abordam especificamente essa teoria.

Tabela 2 - Os primeiros dez artigos com maior número de citações.

\begin{tabular}{l|c}
\multicolumn{1}{c}{ Artigos } & $\begin{array}{c}\text { Nro. Citações } \\
\text { (fonte BD) }\end{array}$ \\
\hline $\begin{array}{l}\text { Human resources management: some new directions (Ferris et } \\
\text { al., 1999) }\end{array}$ & $\begin{array}{c}192 \\
\text { (SCIENCEDIRECT, 2017) }\end{array}$ \\
\hline $\begin{array}{l}\text { Accountability forces in performance appraisal (Klimoski \& } \\
\text { Inks, 1990) }\end{array}$ & 92 \\
\hline $\begin{array}{l}\text { Antecedents and consequences of justice perceptions in } \\
\text { performance appraisals (Erdogan, 2002) }\end{array}$ & (SCIENCEDIRECT, 2017) \\
\hline $\begin{array}{l}\text { The performance evaluation context: Social, emotional, } \\
\text { cognitive, political, and relationship components (Ferris et al., }\end{array}$ & (SCIENCEDIRECT, 2017) \\
\hline
\end{tabular}




\begin{tabular}{l|c}
\hline 2008) & \\
\hline $\begin{array}{l}\text { The accountability of NGOs in civil society and its public } \\
\text { spheres (Lehman, 2007) }\end{array}$ & 31 \\
\hline $\begin{array}{l}\text { Ethical issues in human resources systems (Buckley et al. , } \\
\text { 2001) }\end{array}$ & (SCIENCEDIRECT, 2017) \\
\hline $\begin{array}{l}\text { Using Accountability to Reduce Access Policy Violations in } \\
\text { Information Systems (Vance et al., 2013) }\end{array}$ & 23 \\
\hline $\begin{array}{l}\text { Using accountability to create a more ethical climate (Beu \& } \\
\text { Buckley, 2004) }\end{array}$ & (WEBOFSCIENCE, 2017) \\
\hline $\begin{array}{l}\text { Motivating Knowledge Sharing in Knowledge Management } \\
\text { Systems A Quasi-Field Experiment (Wang et al.,2014) }\end{array}$ & 22 \\
\hline $\begin{array}{l}\text { The planning monitor: } \text { an accountability theory of plan } \\
\text { evaluation (Calkins, 1979) }\end{array}$ & (WEBOFSCIENCE, 2017) \\
\hline For & (WEBOFSCIENCE, 2017) \\
\hline
\end{tabular}

Fonte: Dados da Pesquisa (2017).

A maioria das pesquisas que utilizam os conceitos da AT não é empírica, como demonstrado no Gráfico 3.

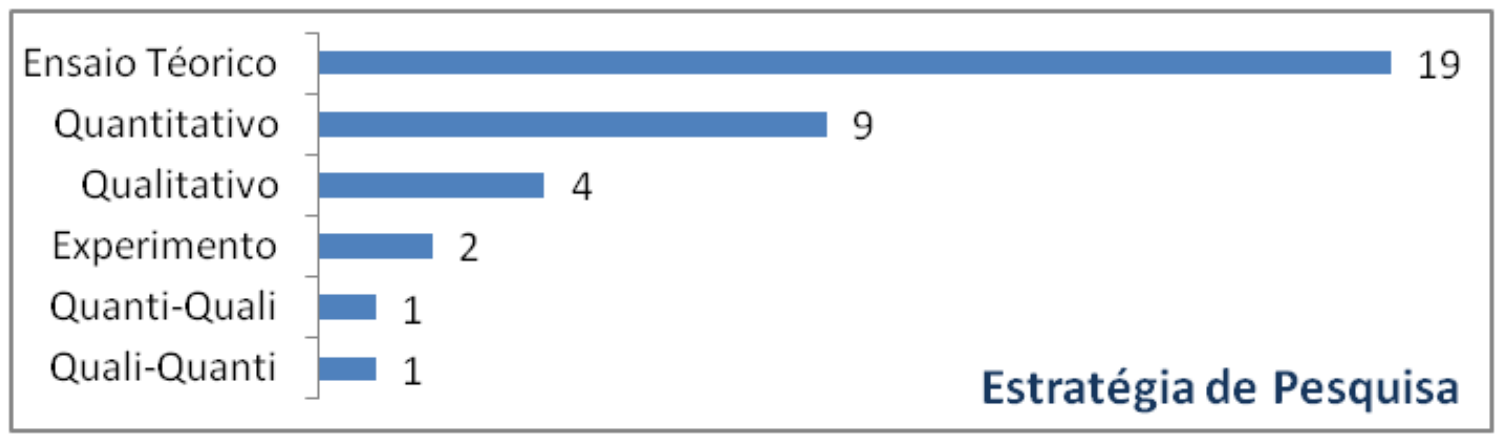

Gráfico 3 - Estratégias de pesquisa de artigos que utilizam conceitos da Accountability Theory. Fonte: Dados da Pesquisa (2017).

Para Shmueli e Koppius (2011), a falta de pesquisas empíricas inviabiliza previsões empíricas e análises preditivas nesses artigos, perdendo poder preditivo. Não obstante, a análise preditiva desempenharia um papel importante na construção teórica e nos testes teóricos, caso as pesquisas fossem empíricas (Shmueli \& Koppius, 2011).

\subsection{Análises das definições e abordagens de pesquisas à AT}

No Gráfico 4, são identificadas as áreas de pesquisa dos artigos sobre AT. Entre os 23 artigos da área de Administração, 11 abrangem a subárea de Recursos Humanos, cinco artigos são da subárea de Sistemas de Informação. Nesta subárea, quatro artigos abordam o tema Segurança da Informação e um aborda o tema Gestão de Conhecimento. 


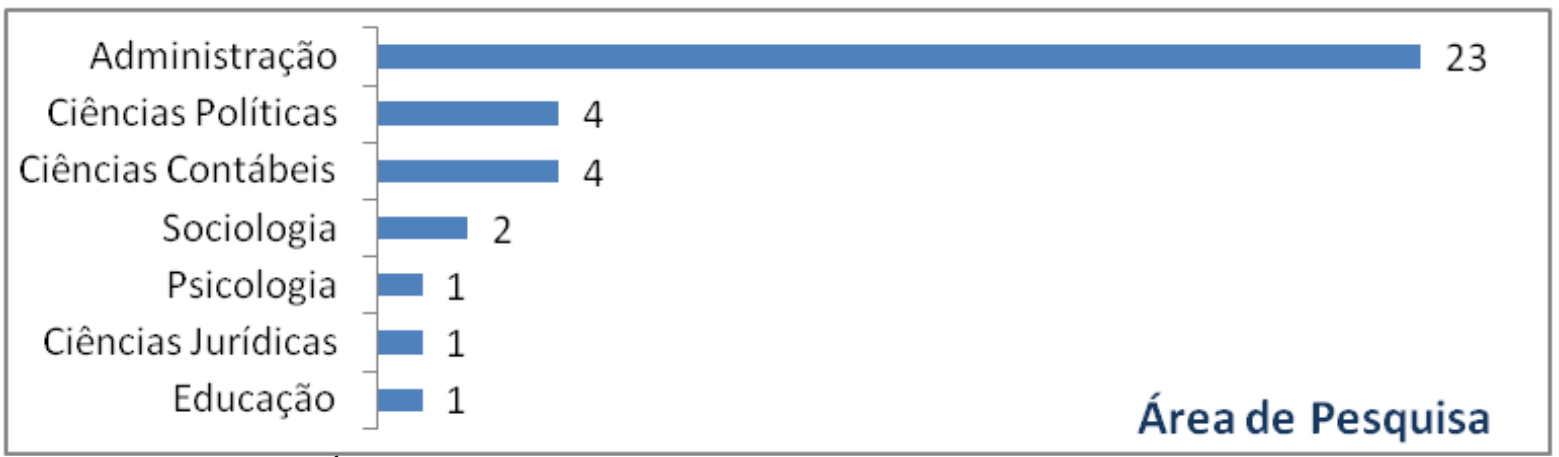

Gráfico 4 - Área de pesquisa de artigos que utilizam conceitos da Accountability Theory. Fonte: Dados da Pesquisa (2017).

No Quadro 1 e 2, são demonstradas evidências de termos utilizados nas definições da AT, obtidos por intermédio da RSL. Esses termos servem para exemplificar a amplitude das definições relativas à AT e seus enfoques de uso. Os termos em negrito destacam aspectos comuns dentre as definições sobre a AT. Os critérios para a seleção das evidências desses quadros foram a clareza e a capacidade de síntese da definição a respeito do conceito accountability na Accountability Theory.

No Quadro 1, estão destacadas as definições do conceito accountability na Accountability Theory e sua relação com a justificativa de comportamento do individuo que é responsabilizado perante os responsabilizadores.

Quadro 1 - Amostragem de evidências de definições sobre o conceito de AT e a relação com a justificativa de comportamento

A accountability na AT é a percepção de defender ou justificar o comportamento de alguém a uma audiência que tenha a autoridade de recompensar ou sancionar, com as recompensas ou sanções sendo percebidas como contingentes à avaliação da audiência (Beu \& Bucley, 2004).

A AT está associada à ética para dirigir o comportamento dos membros da organização. As pessoas geralmente esperam ser responsabilizadas e, em resposta, tentam identificar suas fontes de responsabilidade, em seguida, adaptam o comportamento para acomodar essas responsabilidades. A prestação de contas pode servir como um meio mais eficaz de operacionalizar ou apoiar a cultura ética (Buckley et al., 2001).

A AT refere-se à responsabilidade legal ou ética de prestar contas das ações e atividades a uma pessoa responsável por recebê-las, porém é importante distinguir entre responsibility e accountability. Responsibility refere-se ao dever de agir de uma forma determinada. Accountability é o dever de explicar, justificar ou relatar as ações a alguém e ser responsabilizado pelos resultados das ações (Seay, 2015).

A AT explica como a necessidade percebida de justificar os comportamentos de alguém para outro alguém faz com que se considere e se sinta responsável pelo processo, pelas decisões e julgamentos. Por sua vez, essa necessidade percebida de prestar contas, por uma decisão ou resultado, aumentam a probabilidade de pensar profundamente e sistematicamente sobre os comportamentos processuais (Vance et al., 2015).

Para a prestação de contas, inerente a AT, faz com que as pessoas censurem seu próprio comportamento, quando esperam que outras pessoas revisem suas decisões (Dobbin et al., 2015).

A AT considera o público como um papel ativo na formação dos comportamentos do ator, que está sujeito à responsabilização, sendo que o público é a quem o ator se sente responsável por seu comportamento público. Incluindo dentre esse público o próprio autor (Lee et al., 2012).

As expectativas de responsabilização tornam os funcionários mais dispostos a assumir e apoiar 
ações orientadas para a conformidade, que focaliza principalmente na prevenção, detecção e punição de violações da lei, baseando-se na evasão à sanção (Beu \& Bucley, 2004).

Fonte: Dados da Pesquisa (2017).

No Quadro 2, estão destacadas as definições do conceito accountability na AT e sua relação com o desempenho de indivíduos responsabilizados perante os responsabilizadores.

Quadro 2 - Amostragem de evidências de definições sobre o conceito de AT e a relação com o desempenho com indivíduos responsabilizados.

Uma organização pode responsabilizar os indivíduos pelo seu comportamento na avaliação do desempenho. A avaliação aumenta o desempenho, a eficácia e fornece aos supervisores a oportunidade de avaliar subordinados, comparando seus comportamentos com metas ou padrões estabelecidos. O sistema de avaliação de desempenho é um mecanismo formal, pelo qual os supervisores podem responsabilizar os subordinados, porém também os supervisores podem ser responsabilizados pela organização devido à qualidade de suas avaliações (Ferris et al., 2008).

A accountability na AT é a prestação de contas que implica em ser responsável perante as audiências, por cumprir padrões prescritos, obrigações, deveres, expectativas e outros encargos (Guidice et al., 2016).

Os funcionários que percebem a alta responsabilidade por tarefas de segurança têm maior probabilidade de compartilhar conselhos de segurança e solucionar problemas com outras pessoas (Dang-Pham et al., 2016).

A prestação de contas pode funcionar como um desafio, aumentando o desempenho, ou como uma ameaça, levando a um comportamento autoprotetor e a um desempenho inferior. Indivíduos que esperam cumprir as expectativas comportamentais provavelmente gastarão maior esforço e perseverarão em face da dificuldade, enquanto os que duvidam de sua capacidade de sucesso gastarão menos esforço e abandonarão a tarefa em face da dificuldade (Erdogan et al., 2004).

A AT como uma avaliação justa do desempenho pode ser um mecanismo para aumentar a responsabilidade pela manutenção e melhoria do desempenho (Erdogan, 2002).

No processo de responsabilização corporativa, os responsabilizadores (acionistas) podem exercer influência desafiando ou apoiando a decisão dos responsabilizáveis (administração) sobre práticas e políticas corporativas. Os administradores (responsabilizáveis) têm o desempenho influenciado por acionistas institucionais (responsabilizadores) no diálogo sobre decisões estratégicas corporativas (Uche et al., 2016).

Fonte: Dados da Pesquisa (2017).

Em síntese, conforme as evidências em negrito nos Quadros 1 e 2, a AT aborda a responsabilidade legal ou ética para dirigir e justificar o comportamento de indivíduos, tornando-os responsáveis em cumprir legislações, obrigações, deveres, padrões, metas ou expectativas, perante audiências, que tenham a autoridade de recompensá-los ou sancioná-los. Essa prestação de contas modela o comportamento e influencia positivamente no desempenho desses indivíduos. Essa síntese é reforçada pela nuvem de palavras da Figura 2, na qual são demonstradas as 50 palavras com maior frequência nos artigos que abordam a AT, excetuando elementos gramaticais e numerais. A nuvem de palavras também destaca uma grande preocupação dentre as pesquisas analisadas referentes à gestão (management) dessa prestação de contas e a influência dela no desempenho (performance) organizacional e social dos indivíduos sujeitos à responsabilização. 


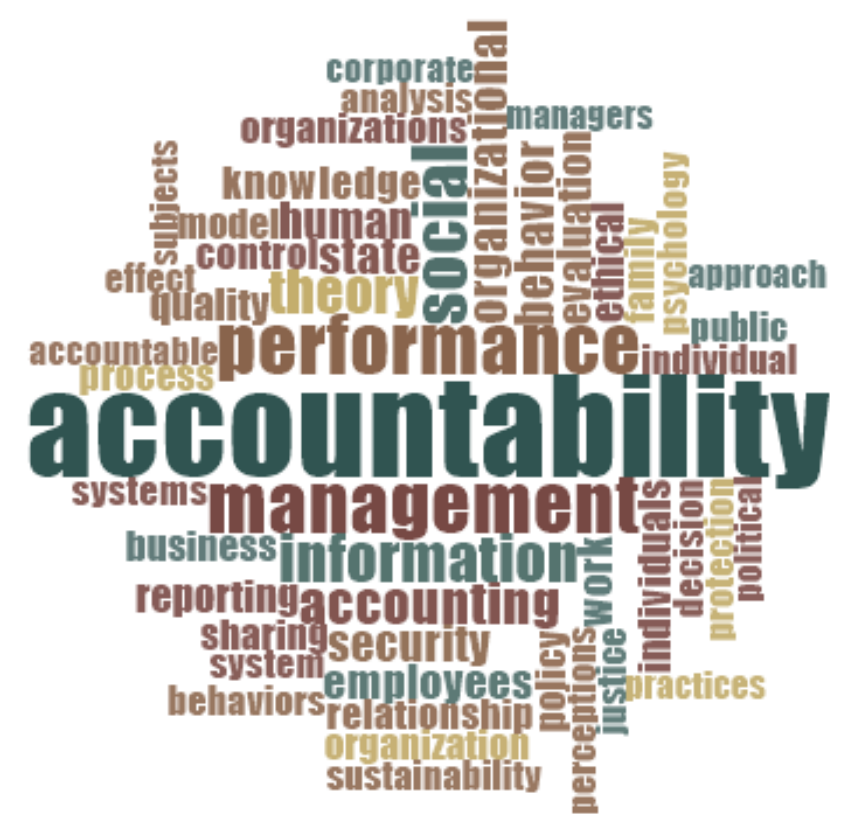

Figura 2 - 50 palavras mais frequentes nos artigos que utilizaram a Accountability Theory.

Fonte: Dados da Pesquisa (2017).

Para responder a questão de pesquisa, na sexta fase da pesquisa foi realizada a categorização dos artigos, na qual foram realizadas buscas nos artigos utilizando os códigos de cada categoria axial de enfoque. Os códigos axiais definidos a priori (Bardin, 2011) foram: transparency, government, corruption, participation, open data, open government e termos correlatos, obtidos a partir das pesquisas de Hossain et al. (2016) e Attard et al. (2015). Dessa forma, dentre os 36 artigos analisados que utilizam conceitos da Accountability Theory (AT) em diversas áreas, 19 artigos abordavam o uso de conceitos da AT relacionados a governo (government), transparência (transparency) ou corrupção (corruption), os quais estão identificados no Quadro 3.

Quadro 3 - Artigos relacionados às categorias de análise Governo, Transparência e Corrupção.

\begin{tabular}{|l|l|}
\hline \multicolumn{1}{|c|}{ Categoria de análise } & \multicolumn{1}{c|}{ Referência } \\
\hline Government (14 artigos) & $\begin{array}{l}\text { Ankamah (2016); Chaney (2013); Chung e Parker (2010); Dobbin et } \\
\text { al. (2015); Ferris et al. (1999); Kalev (2014); Kreander et al. (2009); } \\
\text { Lehman (2013); Lehman (2007); } \\
\text { Shortt e Macdonald (2002); Tusalem (2016); } \\
\text { Vance et al. (2013); Vance et al. (2015); Wilsher (2003). }\end{array}$ \\
\hline Transparency (3 artigos) & $\begin{array}{l}\text { Dobbin et al. (2015); Lehman (2007); } \\
\text { Shortt e Macdonald (2002). }\end{array}$ \\
\hline Corruption (2 artigos) & Ankamah (2016); Tusalem (2016). \\
\hline
\end{tabular}

Fonte: Dados da Pesquisa (2017).

Os artigos sobre AT na categoria de análise Government analisaram a qualidade da prestação de contas governamentais, seus impactos e opções legais, sociais e tecnológicas para a ampliação e qualificação da prestação de contas à sociedade. As evidências destacadas no Quadro 4 foram selecionadas por ilustrar mais claramente e de forma sintética a utilização de conceitos da AT em artigos da categoria government. Nesse quadro, estão destacados em 
negrito os termos que melhor evidenciam os efeitos dos conceitos da AT na área de governo, segundo os artigos analisados.

Quadro 4 - Amostragem de evidências relacionadas às categorias de análise Government.

A prestação de contas é vista como uma qualidade na qual uma pessoa demonstra vontade de aceitar responsabilidade, uma característica desejável em funcionários públicos, agências governamentais ou empresas (Vance et al. 2013).

A informação aumenta a transparência do governo, tornando-a responsável perante aqueles a quem a informação é fornecida. A existência de legislação efetiva de acesso à informação também é essencial para prover o conhecimento necessário para julgar o desempenho do governo.[...] Uma variedade de contratos legais é implementada pelo governo para garantir a prestação de contas, incluindo serviços pessoais baseados no desempenho. Contratos de desempenho destinados a permitir aos gestores das empresas públicas maior autonomia sem sacrificar a prestação de contas e acordos de parceria... Alguns governos consideram a acreditação como forma de prestação de contas na medida em que uma fonte externa atesta a qualidade das atividades de uma instituição (Shortt \& Macdonald, 2002).

Nos últimos 20 anos as sociedades ocidentais testemunharam tendências governamentais que dependem da privatização e da terceirização de funções que antes eram o escopo do setor público na satisfação dos objetivos de prestação de contas. Durante este período, as organizações não governamentais (ONGs) tornaram-se proeminentes na tentativa de preencher o vácuo de responsabilização, expandindo-se para fornecer importantes serviços públicos (Lehman, 2007).

Um princípio fundamental da democracia é que os cidadãos têm o direito e a responsabilidade de exigir a prestação de contas e assegurar que o governo atue no melhor interesse do povo. Os atores públicos podem e devem ser responsabilizados por sua conduta e desempenho [...]. A pressão sobre o governo para promulgar uma lei que tornasse obrigatórios os processos judiciais contra funcionários corruptos e institucionalizasse as audiências públicas, possibilitou o acesso a informações de contas públicas nas auditorias coletivas e possibilitou a investigação de funcionários públicos considerados corruptos (Ankamah, 2016).

Fonte: Dados da Pesquisa (2017).

Os artigos da categoria Transparency destacaram os efeitos entre a transparência na ampliação da prestação de contas governamentais, para melhorar a avaliação e o planejamento de políticas, para proporcionar uma proximidade e ampliar debates democráticos com a sociedade, favorecer o monitoramento e melhorar os efeitos das reformas burocráticas, conforme evidenciado através dos termos em negrito que constam no Quadro 5. Nesse quadro, estão descritos os excertos que mais claramente demonstram a relação entre transparência e conceitos da AT, obtidos a partir dos três artigos dessa categoria de análise. 
Quadro 5 - Amostragem de evidências relacionadas às categorias de análise Transparency.

Os princípios de prestação de contas incluem a abertura, a transparência e a proximidade com a comunidade, na medida em que afetam os debates da sociedade civil... Outro aspecto do papel das ONGs é que elas podem preencher uma lacuna no debate democrático e, assim, promover uma sociedade menos dividida. Ao mesmo tempo, é incluída a responsabilização, para promover a proximidade e a transparência. Se estes princípios puderem ser avançados e satisfeitos, é possível que as ONGs possam alinhar os seus objetivos com os objetivos mais amplos da sociedade. (Lehman, 2007).

Diante da aparente erosão da fé no governo, os políticos estão conscientes da necessidade de transparência no processo de tomada de decisão. Envolver os consumidores nos aspectos de planejamento e avaliação das políticas e pesquisas de saúde é um exemplo de um mecanismo destinado a aumentar a responsabilidade pública (Shortt \& Macdonald, 2002).

Baseando-se em teorias de transparência e prestação de contas, afirmamos que as reformas burocráticas aumentam a transparência para os candidatos a emprego [...]. A responsabilidade conduz os gestores a estarem mais atentos aos efeitos das reformas, tornando mais eficazes as reformas do controle discricionário e da transparência (Dobbin et al., 2015).

Fonte: Dados da Pesquisa (2017).

Dawes (2010) destaca dois princípios da transparência, a utilidade (usefulness) e a garantia (stewardship). Segundo esses princípios, as informações governamentais disponibilizadas precisam estar adequadas ao propósito e ao uso.

Dessa forma, a transparência não é o propósito final, mas um meio, uma forma de disponibilizar dados que atendam a um propósito (Ball, 2009). No contexto das pesquisas analisadas a transparência teve o propósito de prestação de contas e responsabilização, consoante à accountability com um processo da Accountability Theory.

Em relação à adequação ao uso, De Ferranti et al. (2009) indica que a transparência, em termos governamentais, refere-se à disponibilidade pública e oportuna, com qualidade, abrangente e relevante, de informações confiáveis sobre as atividades do governo.

A síntese e a relação entre os conceitos apresentados nos artigos das categorias de análise que abrangeram Accountability Theory conjuntamente com Government e Transparency estão apresentados na Figura 3. 


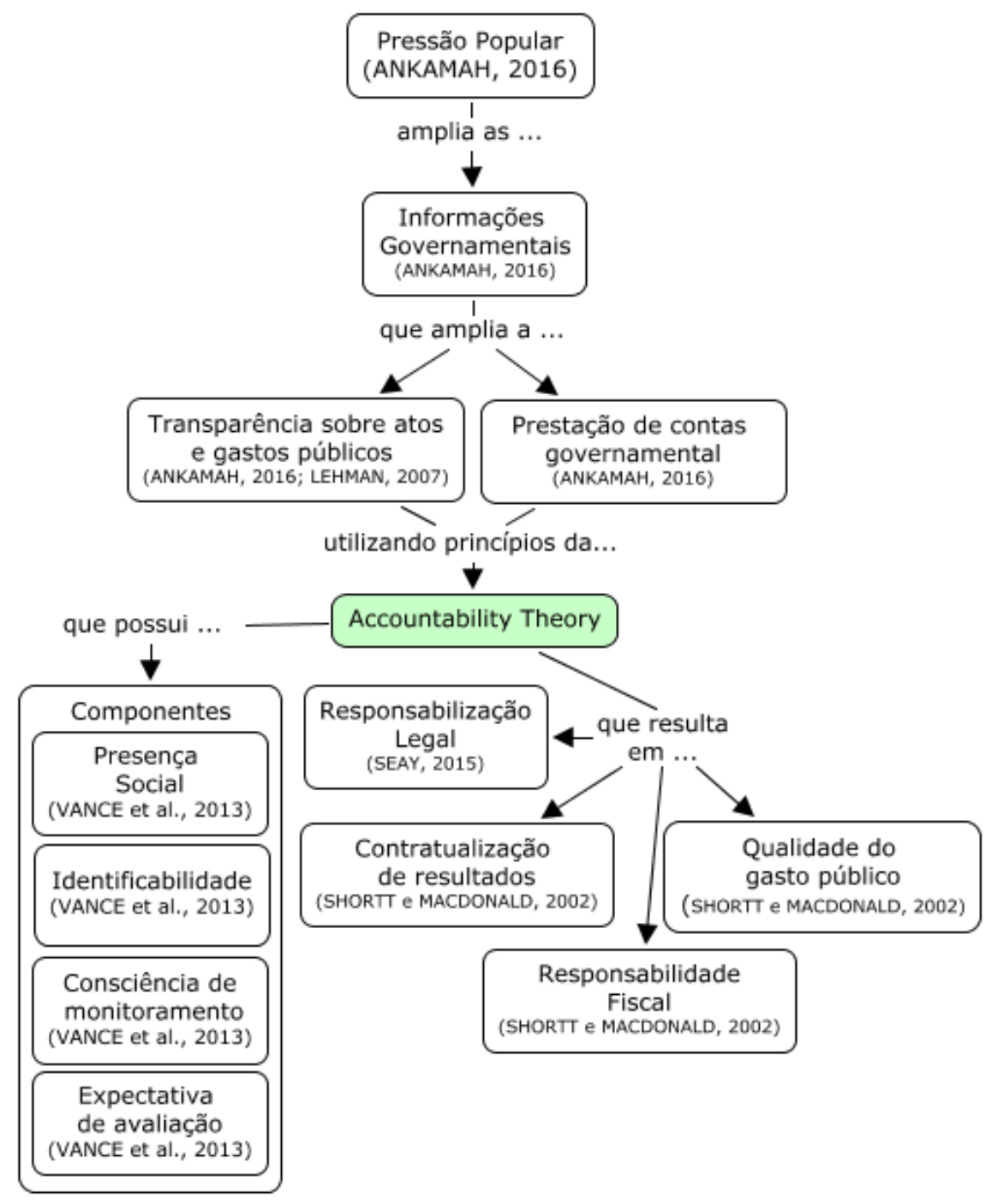

Figura 3 - Mapa conceitual dos artigos sobre Government, Transparency e AT, conforme evidências.

Fonte: Dados da Pesquisa (2017).

\subsection{Perspectivas de ampliação da pesquisa sobre AT na área governamental}

Demais termos como participation, open data, open government foram pesquisados, mas não foram encontrados artigos abordando esses termos. Dessa forma, surpreende a falta de abordagem do uso de dados abertos conjuntamente à AT, por se tratarem de uma importante ferramenta para a redução dos níveis de corrupção ao permitir uma maior transparência em termos de atividades e gastos públicos, tornando-se um elemento importante em várias intervenções de responsabilização (Attard et al., 2015). Nesse sentido, nos últimos anos ocorreu um incremento na disponibilidade de dados abertos, ou seja, dados que podem ser livremente utilizados, modificados e compartilhados por qualquer pessoa para qualquer finalidade (Iglesias, 2017). Como um exemplo dessa aplicação, sugere-se que os quatro componentes fundamentais da AT (Vance et al., 2013), podem assumir os seguintes enfoques em relação aos dados abertos, conforme segue:

a) Identificabilidade: a crença de um agente público em ser identificado, através dos Dados Abertos Governamentais (DAGs); 
b) Expectativa de avaliação: a crença do agente público que seu desempenho será avaliado pela sociedade (cidadãos e entidades organizadas da sociedade civil), de acordo com algumas regras básicas normativas e com consequências contidas na legislação;

c) Consciência de monitoramento: estado da cognição ativo, de um agente público, que considera que o seu trabalho é monitorado pela sociedade através dos DAGs; e

d) Presença social: a consciência da existência de cidadãos utilizando os DAGs, o que potencializa a consciência de monitoramento.

Nesse contexto, o agente público é o indivíduo que exerce função em organizações governamentais, por qualquer forma de investidura ou vínculo, mandato, cargo ou emprego (Brasil, 1992).

Dessa forma, há a perspectiva de os Dados Abertos Governamentais (DAGs) atuarem como um potencializador da prestação de contas e da responsabilização, por intermédio da ampliação da transparência com esse propósito. No entanto, questões como a compreensão, qualidade e a fidedignidade dos DAGs, para ampliação da transparência e com o propósito de atender ao processo de accountability da AT, são especialmente preocupantes, pois apesar da transparência ser um predecessor da accountability (Al-Jamal \& Abu-Shanab, 2016), a relação entre a transparência e a accountability, através dos DAGs, ainda é esporádica, impulsionada por circunstâncias específicas, por questões locais e uso aleatório dos dados (Worthy, 2015). Um dos motivos pode ser a falta de qualidade dos dados, que se caracteriza como uma ameaça à transparência (Koussouris et al., 2015). Como exemplo, no Reino Unido não foi atingido o pleno objetivo de accountability por intermédio da transparência, devido à falta de participação do cidadão ocasionada pela falta de compreensão e confiança nos dados (Barry \& Bannister, 2014).

\section{Considerações Finais}

As expectativas de responsabilização tornam os funcionários mais dispostos a assumir e apoiar ações orientadas para a conformidade, quando visa à prevenção, detecção e punição de violações de leis e regras. Os resultados demonstraram uma potencial área de pesquisa empírica sobre o uso da AT, abrangendo a transparência e o combate à corrupção, pois a prestação de contas, e a posterior responsabilização dos agentes públicos envolvidos, são inerentes à legislação sobre controle dos gastos e ações governamentais.

Dentre os usos da Accountability Theory (AT), destacou-se entre os artigos analisados o reiterado uso de mecanismos de prestação de contas e responsabilização na avaliação de desempenho de funcionários e a relação do uso eficaz desses mecanismos à melhoria do desempenho dos funcionários. Paralelamente, a AT foi utilizada ainda em artigos que abordaram prestações de contas de ONGs, relatórios de sustentabilidade e conformidade com políticas. A quantidade de artigos que abordam ou que utilizam a AT foi inferior ao esperado, apesar dessa teoria não ser recente. No entanto, pesquisas em outras bases de dados bibliográficas ou em outros idiomas podem trazer um panorama diferente.

Considerando as pesquisas que utilizaram elementos e componentes da AT no contexto social e organizacional, há um grande potencial de ampliação desse uso dessa teoria na responsabilização de funcionários públicos e no combate à corrupção, utilizando aos Dados Abertos Governamentais (DAG) como um mecanismo de disponibilização de informações à prestação de contas. Nesse contexto, o DAG, oriundo do Governo Aberto, promove a transparência através da publicação de dados do governo, possibilitando ao cidadão ver o que o governo faz e permitindo a responsabilização dos agentes públicos por suas ações e decisões 
tomadas, contribuindo no processo de accountability da AT, além de divulgar informações governamentais que podem ser reutilizadas e proporcionem valor social e econômico.

Dessa forma, os resultados demonstraram que há uma potencial área de pesquisa empírica sobre o uso da AT a ser explorada, abrangendo transparência, combate à corrupção, dados abertos e governo aberto, pois a prestação de contas e a posterior responsabilização são inerentes à legislação sobre controle dos gastos e ações governamentais. Os Dados Abertos Governamentais, por sua vez, são o instrumento para disponibilizar as informações a serem prestadas à sociedade e podem se tornar uma ferramenta de empoderamento do cidadão na redução dos níveis de corrupção, ajudando os cidadãos a aprender sobre as atividades governamentais, melhorar a accountability governamental e permitir que os cidadãos participem do processo político. Todavia, o DAG pode auxiliar na mitigação da corrupção, aumentando a prestação de contas e fortalecendo a democracia, porque os eleitores podem tomar decisões estando mais bem informados, e um público bem informado e confiante também fortalece o próprio governo, pois é mais provável que se comprometa com iniciativas governamentais. Nesse sentido, é especialmente importante à realização de pesquisas que mensurem a efetividade da responsabilização dos agentes públicos e o aumento do desempenho desses indivíduos, sujeitos à responsabilização, em um contexto governamental.

\section{REFERÊNCIAS}

Akutsu, L., \& Pinho, J. A. G. (2002). Sociedade da informação, accountability e democracia delegativa: investigação em portais de governo no Brasil. Revista de Administração Pública, 36(5), 723-745.

Al-Jamal, M., \& Abu-Shanab, E. (2016). The influence of open government on e-government website: the case of Jordan. International Journal of Electronic Governance, 8(2), 159179.

Andersen, T. B. (2009). E-Government as an anti-corruption strategy. Information Economics and Policy, 21(3), 201-210.

Ankamah, S. S. (2016). Toward a framework of improving horizontal and social accountability mechanisms: Case studies in Asia. Asian Education and Development Studies, 5(3), 288-304.

Attard, J., Orlandi, F., Scerri, S., \& Auer, S. (2015). A systematic review of open government data initiatives. Government Information Quarterly, 32(4), 399-418.

Ball, C. (2009). What is transparency? Public Integrity, 11(4), 293-308.

Bardin, L. (2011). Análise de Conteúdo 70 ed. São Paulo: Almedina Brasil.

Barkemeyer, R., Comyns, B., Figge, F., \& Napolitano, G. (2014, December). CEO statements in sustainability reports: Substantive information or background noise?. In Accounting Forum (Vol. 38, No. 4, pp. 241-257). Elsevier.

Barry, E., \& Bannister, F. (2014). Barriers to open data release: A view from the top. Information Polity, 19(1, 2), 129-152.

Baumeister, R. F., \& Leary, M. R. (1997). Writing narrative literature reviews. Review of general psychology, 1(3), 311. 
Bem, D. J. (1995). Writing a review article for Psychological Bulletin. Psychological Bulletin, 118(2), 172.

Beu, D. S., \& Buckley, M. R. (2004). Using accountability to create a more ethical climate. Human resource management review, 14(1), 67-83.

Brasil. Lei $\mathrm{N}^{\circ} 8.429$, de 2 de junho de 1992. Dispõe sobre as sanções aplicáveis aos agentes públicos nos casos de enriquecimento ilícito no exercício de mandato, cargo, emprego ou função na administração pública direta, indireta ou fundacional e dá outras providências. Diário Oficial da União, Brasília, DF, 3 de junho de 1992.

Buckley, M. R., Beu, D. S., Frink, D. D., Howard, J. L., Berkson, H., Mobbs, T. A., \& Ferris, G. R. (2001). Ethical issues in human resources systems. Human resource management review, 11(1-2), 11-29.

Calkins, H. W. (1979). The planning monitor: an accountability theory of plan evaluation. Environment and Planning A, 11(7), 745-758.

Campos, A. M. (1990). Accountability: quando poderemos traduzi-la para o português?. Revista de administração pública, 24(2), 30-50.

Chaney, P. (2013). Electoral Discourse Analysis of Civil Conflict Resolution: The Case of Northern Ireland in UK Statewide Elections 1970-2010. Irish political studies, 28(1), 119.

Chung, L. H., \& Parker, L. D. (2010, March). Managing social and environmental action and accountability in the hospitality industry: A Singapore perspective. In Accounting Forum (Vol. 34, No. 1, pp. 46-53). Elsevier.

Comyns, B., Figge, F., Hahn, T., \& Barkemeyer, R. (2013, September). Sustainability reporting: The role of "search","experience" and "credence" information. In Accounting Forum (Vol. 37, No. 3, pp. 231-243). Elsevier.

Cooper, H., Hedges, L. V., \& Valentine, J. C. (Eds.). (2009). The handbook of research synthesis and meta-analysis. Russell Sage Foundation.

Dang-Pham, D., Pittayachawan, S., \& Bruno, V. (2016). Impacts of security climate on employees' sharing of security advice and troubleshooting: Empirical networks. Business Horizons, 59(6), 571-584.

Dang-Pham, D., Pittayachawan, S., \& Bruno, V. (2017). Why employees share information security advice? Exploring the contributing factors and structural patterns of security advice sharing in the workplace. Computers in Human Behavior, 67, 196-206.

Dawes, S. S. (2010). Stewardship and usefulness: Policy principles for information-based transparency. Government Information Quarterly, 27(4), 377-383.

De Ferranti, D., Jacinto, J., Ody, A. J., \& Ramshaw, G. (2009). How to improve governance: a new framework for analysis and action. Brookings Institution Press.

Dobbin, F., Schrage, D., \& Kalev, A. (2015). Rage against the iron cage: The varied effects of bureaucratic personnel reforms on diversity. American Sociological Review, 80(5), 1014-1044.

Erdogan, B. (2002). Antecedents and consequences of justice perceptions in performance appraisals. Human resource management review, 12(4), 555-578. 
Erdogan, B., Sparrowe, R. T., Liden, R. C., \& Dunegan, K. J. (2004). Implications of organizational exchanges for accountability theory. Human Resource Management Review, 14(1), 19-45.

Ferris, G. R., Hochwarter, W. A., Buckley, M. R., Harrell-Cook, G., \& Frink, D. D. (1999). Human resources management: Some new directions. Journal of management, 25(3), 385-415.

Ferris, G. R., Munyon, T. P., Basik, K., \& Buckley, M. R. (2008). The performance evaluation context: Social, emotional, cognitive, political, and relationship components. Human Resource Management Review, 18(3), 146-163.

Flick, U. (2009). Introdução a Pesquisa Qualitativa. 3 ed. Porto Alegre:Artmed.

Franzen, L., Meckfessel, M., Moehrle, S. R., \& Reynolds-Moehrle, J. A. (2015). Developments in accounting regulation: A synthesis and annotated bibliography of evidence and commentary in the 2012 academic literature. Research in Accounting Regulation, 27(1), 21-38.

Guidice, R. M., Mero, N. P., \& Greene, J. V. (2013). Perceptions of accountability in family business: Using accountability theory to understand differences between family and nonfamily executives. Journal of Family Business Strategy, 4(4), 233-244.

Guidice, R. M., Mero, N. P., Matthews, L. M., \& Greene, J. V. (2016). The influence of individual regulatory focus and accountability form in a high performance work system. Journal of Business Research, 69(9), 3332-3340.

Harrison, T. M., Guerrero, S., Burke, G. B., Cook, M., Cresswell, A., Helbig, N., ... \& Pardo, T. (2012). Open government and e-government: Democratic challenges from a public value perspective. Information Polity, 17(2), 83-97.

Höffner, K., Martin, M., \& Lehmann, J. (2016). LinkedSpending: openspending becomes linked open data. Semantic Web, 7(1), 95-104.

Hossain, M. A., Dwivedi, Y. K., \& Rana, N. P. (2016). State-of-the-art in open data research: Insights from existing literature and a research agenda. Journal of organizational computing and electronic commerce, 26(1-2), 14-40.

Iglesias, D. Open Data And The Fight Against Corruption In Brazil. Transparency International, $2017 . \quad$ Disponível em: $<$ https://www.transparency.org/whatwedo/publication/ open_data_and_the_fight_against_corruption_in_brazil>. Acesso em: $27 \mathrm{fev} .2017$.

Janssen, K. (2011). The influence of the PSI directive on open government data: An overview of recent developments. Government Information Quarterly, 28(4), 446-456.

Kalev, A. (2014). How you downsize is who you downsize: Biased formalization, accountability, and managerial diversity. American Sociological Review, 79(1), 109135.

Klimoski, R., \& Inks, L. (1990). Accountability forces in performance appraisal. Organizational Behavior and Human Decision Processes, 45(2), 194-208. 
Koussouris, S., Lampathaki, F., Kokkinakos, P., Askounis, D., \& Misuraca, G. (2015). Accelerating Policy Making 2.0: Innovation directions and research perspectives as distilled from four standout cases. Government Information Quarterly, 32(2), 142-153.

Kreander, N., Beattie, V., \& McPhail, K. (2009). Putting our money where their mouth is: alignment of charitable aims with charity investments-tensions in policy and practice. The British Accounting Review, 41(3), 154-168.

Lee, M. J., Kim, S. S., \& Kim, W. (2012). Service Employees' Organizational Citizenship Behaviors and Customer-Oriented Behaviors: An Accountability Theory Perpective. Journal of foodservice business research, 15(4), 335-361.

Lehman, G. (2013). Critical reflections on Laughlin's middle range research approach: Language not mysterious?. Critical Perspectives on Accounting, 24(3), 211-224.

Lehman, G. (2007). The accountability of NGOs in civil society and its public spheres. Critical Perspectives on Accounting, 18(6), 645-669.

Lerner, J. S., \& Tetlock, P. E. (1999). Accounting for the effects of accountability. Psychological bulletin, 125(2), 255.

Lourenço, R. P. (2015). An analysis of open government portals: A perspective of transparency for accountability. Government Information Quarterly, 32(3), 323-332.

Murillo, M. J. (2015). Evaluating the role of online data availability: The case of economic and institutional transparency in sixteen Latin American nations. International Political Science Review, 36(1), 42-59.

O'Leary-Kelly, A. M., Tiedt, P., \& Bowes-Sperry, L. (2004). Answering accountability questions in sexual harassment: Insights regarding harassers, targets, and observers. Human Resource Management Review, 14(1), 85-106.

Pinho, J. A. G. D., \& Sacramento, A. R. S. (2009). Accountability: can we now translate it into Portuguese?. Revista de Administração Pública, 43(6), 1343-1368.

Raupp, F. M., \& de Pinho, J. A. G. (2013). Accountability em câmaras municipais: uma investigação em portais eletrônicos. Revista de administração, 48(4), 770-782.

SCIENCEDIRECT. Disponível em: 〈http://www.sciencedirect.com>. Acesso em: 06 fev. 2017.

Schillemans, T., Van Twist, M., \& Vanhommerig, I. (2013). Innovations in accountability: Learning through interactive, dynamic, and citizen-initiated forms of accountability. Public Performance \& Management Review, 36(3), 407-435.

SCOPUS. Disponível em: <http://www.scopus.com>. Acesso em: 06 fev. 2017.

Seay, S. S. (2015). Sustainability is applied ethics. Journal of Legal, Ethical and Regulatory Issues, 18(2), 63.

Shmueli, G., \& Koppius, O. R. (2011). Predictive analytics in information systems research. Mis Quarterly, 553-572.

Shortt, S. E. D., \& Macdonald, J. K. (2002, June). Toward an accountability framework for Canadian healthcare. In Healthcare Management Forum (Vol. 15, No. 2, pp. 24-32). No longer published by Elsevier. 
Sol, D. A. D. (2013). The institutional, economic and social determinants of local government transparency. Journal of Economic Policy Reform, 16(1), 90-107.

Stamati, T., Papadopoulos, T., \& Anagnostopoulos, D. (2015). Social media for openness and accountability in the public sector: Cases in the Greek context. Government Information Quarterly, 32(1), 12-29.

Tetlock, P. E. (1983a). Accountability and complexity of thought. Journal of personality and social psychology, 45(1), 74.

Tetlock, P. E. (1983b). Accountability and the perseverance of first impressions. Social Psychology Quarterly, 285-292.

Tetlock, P. E. (1985). Accountability: A social check on the fundamental attribution error. Social Psychology Quarterly, 227-236.

Tetlock, P. E., \& Kim, J. I. (1987). Accountability and judgment processes in a personality prediction task. Journal of personality and social psychology, 52(4), 700.

Tetlock, P. E., \& Boettger, R. (1989). Accountability: A social magnifier of the dilution effect. Journal of personality and social psychology, 57(3), 388.

Tetlock, P. E., Skitka, L., \& Boettger, R. (1989). Social and cognitive strategies for coping with accountability: conformity, complexity, and bolstering. Journal of personality and social psychology, 57(4), 632.

Tetlock, P. E., \& Boettger, R. (1994). Accountability amplifies the status quo effect when change creates victims. Journal of Behavioral Decision Making, 7(1), 1-23.

Tober, M. (2011). PubMed, ScienceDirect, Scopus or Google Scholar-Which is the best search engine for an effective literature research in laser medicine?. Medical Laser Application, 26(3), 139-144.

Tusalem, R. F. (2016). Political and economic accountability in a delegative democracy. International Political Science Review, 37(4), 517-532.

Uche, C. O., Adegbite, E., \& Jones, M. (2016, June). Institutional shareholder activism in Nigeria: An accountability perspective. In Accounting Forum (Vol. 40, No. 2, pp. 7888). Elsevier.

Vance, A., Lowry, P., \& Eggett, D. (2015). Increasing Accountability through the User Interface Design Artifacts: A New Approach to Addressing the Problem of AccessPolicy Violations. MIS quarterly, 39(2), 345-366.

Vance, A., Lowry, P. B., \& Eggett, D. (2013). Using accountability to reduce access policy violations in information systems. Journal of Management Information Systems, 29(4), 263-290.

Wang, S., Noe, R. A., \& Wang, Z. M. (2014). Motivating knowledge sharing in knowledge management systems: A quasi-field experiment. Journal of Management, 40(4), 9781009.

Wang, Q., \& Waltman, L. (2016). Large-scale analysis of the accuracy of the journal classification systems of Web of Science and Scopus. Journal of Informetrics, 10(2), 347-364. 
WEB OF KNOWLEDGE. Disponível em: <http://apps.webofknowledge.com>. Acesso em: 06 fev. 2017.

Wilsher, D. (2003). Non-State Actors and the Definition of a Refugee in the United Kingdom: Protection, Accountability or Culpability?. international Journal of Refugee law, 15(1), 68-112.

Worthy, B. (2015). The impact of open data in the UK: Complex, unpredictable, and political. Public Administration, 93(3), 788-805. 